\title{
Diagnostic benefits of presurgical fMRI in patients with brain tumours in the primary sensorimotor cortex
}

\author{
Martina Wengenroth • M. Blatow • J. Guenther • \\ M. Akbar • V. M. Tronnier • C. Stippich
}

Received: 9 September 2010 /Revised: 2 November 2010 /Accepted: 8 December 2010 /Published online: 28 January 2011

(C) The Author(s) 2011. This article is published with open access at Springerlink.com

\begin{abstract}
Objectives Reliable imaging of eloquent tumour-adjacent brain areas is necessary for planning function-preserving neurosurgery. This study evaluates the potential diagnostic benefits of presurgical functional magnetic resonance imaging (fMRI) in comparison to a detailed analysis of morphological MRI data.

Methods Standardised preoperative functional and structural neuroimaging was performed on 77 patients with rolandic mass lesions at 1.5 Tesla. The central region of both hemispheres was allocated using six morphological and three functional landmarks.

Results fMRI enabled localisation of the motor hand area in 76/77 patients, which was significantly superior to analysis of structural MRI (confident localisation of motor hand area
\end{abstract}

M. Wengenroth $(\bowtie) \cdot$ M. Blatow $\cdot$ J. Guenther

Department of Neuroradiology,

University of Heidelberg Medical School,

Im Neuenheimer Feld 400,

69120 Heidelberg, Germany

e-mail: martina.wengenroth@med.uni-heidelberg.de

\section{Akbar}

Department of Orthopaedics,

University of Heidelberg Medical School,

Schlierbacher Landstraße 200a,

69118 Heidelberg, Germany

\section{M. Tronnier}

Department of Neurosurgery, University of Schleswig-Holstein, Campus Luebeck, Ratzeburger Allee 160,

23538 Luebeck, Germany

\section{Stippich}

Department of Diagnostic and Interventional Neuroradiology,

University Hospital Basle,

Petersgraben 4,

CH 4031 Basle, Switzerland in 66/77 patients; $p<0.002$ ). FMRI provided additional diagnostic information in $96 \%$ (tongue representation) and $97 \%$ (foot representation) of patients. FMRI-based presurgical risk assessment correlated in $88 \%$ with a positive postoperative clinical outcome.

Conclusion Routine presurgical FMRI allows for superior assessment of the spatial relationship between brain tumour and motor cortex compared with a very detailed analysis of structural 3D MRI, thus significantly facilitating the preoperative risk-benefit assessment and function-preserving surgery. The additional imaging time seems justified. FMRI has the potential to reduce postoperative morbidity and therefore hospitalisation time.

Keywords Presurgical functional magnetic resonance imaging - Central region · Brain tumours · Motor hand area . Hand knob · Dynamic threshold

\section{Introduction}

The central region of the brain comprises the central sulcus and the pre- and postcentral gyri, functionally corresponding to the primary motor (M1, Brodmann area 4) and somatosensory (S1, Brodmann areas 1-3) cortices. Hence, neurosurgery involving the central region, e.g. for tumour removal, can lead to both motor and somatosensory deficits alike. While radical brain tumour resection yields higher survival rates, it is unfortunately associated with increased postoperative morbidity [1]. In patients with aggressive and infiltrative tumour growth and thus a low prospect of curative treatment it is particularly important to avoid surgery-induced impairments. Instead, emphasis should be placed on ensuring life quality by preserving neurological function. In these cases neurosurgical operations require strict indication weighed up 
against alternative and less invasive therapeutic strategies. Therefore, additional information concerning structure and functionality of affected brain regions is necessary for careful consideration and planning of neurosurgical interventions.

Magnetic resonance imaging (MRI) is the established standard imaging technique for brain tumours in particular because of its high-resolution multiplanar images, excellent soft tissue contrast and lack of ionising radiation. For identification of the central region several morphological landmarks are being used, however, their reliability is often compromised by large inter-individual anatomical variability, even in healthy subjects [2]. The most robust morphological landmark is the motor hand area, which represents as a characteristic dorsally oriented convexity in the precentral gyrus, the so-called "hand knob" on transverse planes or "hook sign" on sagittal planes [3-6] (Fig. 1). Other representations of the human body lack such distinct morphological correlates [7], hence their localisation is restricted to a rough estimate based on the somatotopic organisation of the primary motor and somatosensory cortices (homunculus) [8]. In addition, diagnostic inaccuracy is often prompted by infiltrative and displacing mass lesions and pathological signal alterations involving the pre- or postcentral gyrus, which can lead to extensive changes of anatomical proportions, partially or completely impeding presurgical neuroimaging. Furthermore, functional areas may be shifted by brain tumours beyond typical landmarks [9-11]. To avoid permanent damage to neurological function, identification of eloquent cortical areas plays a critical role in the presurgical planning of tumour resection [12, 13]. Intra-operative mapping of brain functions by means of electrocorticography $(\mathrm{ECoG})$ or sensory-evoked potential monitoring are still considered the reference techniques [12]. However, contrary to these invasive techniques, functional magnetic resonance imaging (fMRI) offers such diagnostic information non-invasively before surgery and with justifiable clinical expenditure [13, 14]. fMRI assesses brain activity indirectly by detection of local haemodynamic changes that reflect actual neuronal activity of functional areas. The blood-oxygen-leveldependent (BOLD) technique measures function-dependent decreased desoxyhaemoglobin concentrations of activated brain areas on T2*-weighted images [15-18]. Several studies comparing BOLD-fMRI with established reference methods such as ECoG validated the reliability of presurgical localisation of the primary motor cortex [19-23]. Although presurgical localisation of the motor cortex is the most established clinical application of fMRI nowadays, employed in a continuously increasing number of clinics, there are still no consistent and uniform guidelines regarding data acquisition, analysis and medical interpretation. Addi-

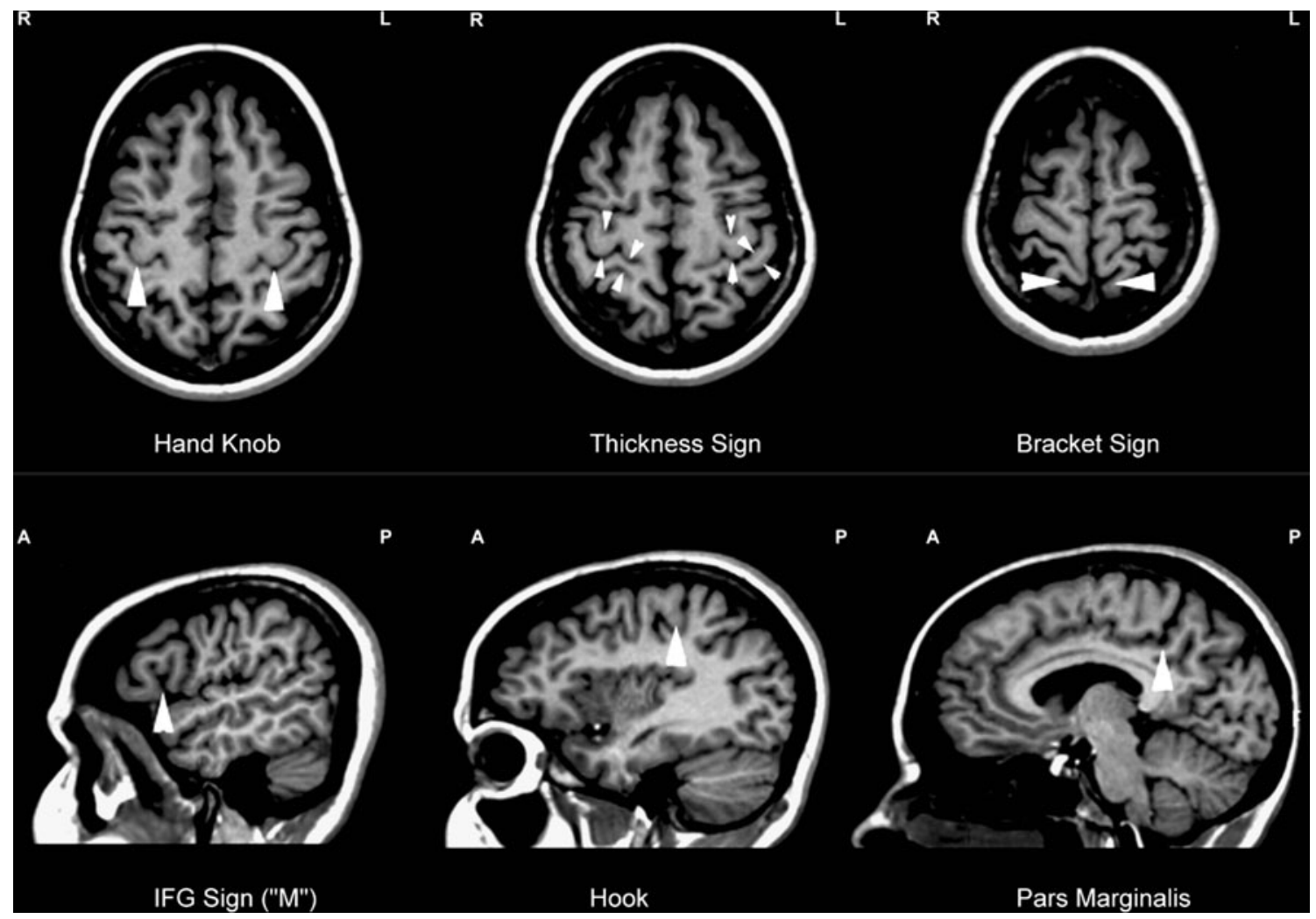

Fig. 1 Anatomical landmarks of the central region (24-year-old woman). Axial sections (top): hand knob (left), thickness sign (middle), bracket sign (right). Sagittal sections (bottom): inferior frontal gyrus (IFG, left), precentral hook (middle), pars marginalis (right). From [43] 
tionally, only a few authors have attempted to analyse the diagnostic value of presurgical fMRI, and existing reports are mainly based on non-standardised investigations in smaller groups of patients [24-28]. The lack of large-scale presurgical fMRI studies is apparent and so far impeded further clinical implementation of this technique within the diagnostic setting prior to neurosurgery [12]. Undoubtedly, it is absolutely essential to critically evaluate diagnostic potential and clinical benefits of such a powerful preoperative imaging technique with standardised methods in a representative group. Accordingly, this study compares diagnostic benefits of presurgical fMRI with those of morphological MRI in a large number of patients with brain tumours of the central region.

\section{Materials and methods}

\section{Subjects}

In the present study 77 consecutive patients (42 female; 35 male, mean age 51 years; range $16-80$ years) with brain tumours of the central region were investigated according to standardised protocols for presurgical investigations with morphological and functional MRI before potential neurosurgical operation. All subjects gave informed consent before the experiment and the protocol was approved by the medical ethics committee of the University of Heidelberg Medical School. Clinical data were obtained retrospectively from operation protocols and medical records. Aetiologies of the mass lesions were heterogeneous, mainly of malignant origin (see Table 1). Tumours were localised in the left hemisphere in 38 patients and in the right in 37 patients; in 2 cases multiple metastases affected both hemispheres. Caused by the mass lesion, 29 patients suffered from motor deficits, 21 from somatosensory deficits and 10 patients had combined motor and somatosensory deficits at the time of imaging. Furthermore, focal and generalised seizures, language and speech deficits, word finding deficits and/or change of personality were observed in 37 patients. Some patients reported only mild impairments such as headache and vertigo. No reliable clinical data were available for 2 subjects retrospectively. In 3 cases patients did not present with any clinical symptoms and mass lesions were detected incidentally ( 1 cavernoma, 2 meningiomas).

\section{Morphological MRI}

All patients were investigated at 1.5 Tesla (Marconi Edge, Cleveland, OH, USA) using a conventional birdcage headcoil. Movement artefacts were reduced by comfortable positioning of the subject and fixing the head with
Table 1 Classification of cerebral mass lesions in the central region of patients included in the study

\begin{tabular}{lr}
\hline Tumour type (WHO grade) & Number \\
\hline Astrocytoma (grade I) & 1 \\
Astrocytoma (grade II) & 7 \\
Astrocytoma (grade III) & 7 \\
Astrocytoma (unknown grade) & 3 \\
Glioblastoma & 19 \\
Gliosarcoma & 2 \\
Oligodendroglioma (grade II) & 2 \\
Oligodendroglioma (grade III) & 1 \\
Oligodendroglioma (unknown grade) & 1 \\
Oligoastrocytoma (grade II) & 2 \\
Meningioma (grade I) & 4 \\
Atypical meningioma (grade II) & 1 \\
Malignant histiocytoma & 1 \\
Metastasis & 17 \\
Angioma/Cavernoma & 7 \\
Brain abscess & 2 \\
Total & 77 \\
\hline
\end{tabular}

preformed foam pads. Subsequent to intravenous administration of gadolinium-containing contrast agent (Magnevist ${ }^{\mathbb{R}}$ or Omniscan ${ }^{\circledR}$, standard dosage: $0.2 \mathrm{ml} / \mathrm{kg}$ ) patients received a T1-weighted 3-D anatomical data set (MPRAGE, TR/TE= $30 / 4.4 \mathrm{~ms}, 135$ axial slices, slice thickness $1.3 \mathrm{~mm}$ ), which was employed for neuronavigation as well as for superimposition of functional data. The diameter of the contrastenhancing perirolandic mass lesions on axial images ranged between 4 and $24 \mathrm{~mm}$.

The central region was identified on T1-weighted structural images according to 6 established morphological landmarks in both hemispheres [5-7, 14, 29-31]. The unaffected hemisphere served as an internal reference for physiological abundance, distribution and anatomical variants of the employed landmarks in this group. The following morphological landmarks for the localisation of the central region were used (see Fig. 1):

- Thickness sign: The precentral gyrus and its cortex layer are typically thicker than the postcentral gyrus.

- Hand knob and hook: The motor hand area in the precentral gyrus protrudes as a posteriorly directed 'omega'- or 'epsilon'-shaped convexity on axial planes as well as a dorsally directed 'hook' on sagittal images.

- Pars marginalis and bracket sign: Both partes marginales of the cingulate sulci are located behind the central sulcus on parasagittal planes. On axial sections both medial subdivisions of the postcentral gyrus form imaginary 'brackets' around the partes marginales of the cingulate sulci. 
- Inferior frontal gyrus: On parasagittal sections the inferior frontal gyrus presents with an M-shaped configuration comprising the partes orbitalis, triangularis and opercularis, the latter being functionally associated with Broca's motor speech area. The sulcus dorsal of the "M" disembogues into the precentral sulcus.

\section{Functional MRI}

All fMRI investigations were performed with standardised block-designed BOLD technique employing a T2*-weighted single-shot, blipped gradient echo Echo-Planar-Imaging sequence (GE-EPI, TR/TE $=4,000 / 80 \mathrm{~ms}, \mathrm{FOV}=256 \times 256 \mathrm{~mm}^{2}$, image matrix $=128 \times 128$ voxels, flip angle $=90^{\circ}, 22$ contiguous axial images, slice thickness $5 \mathrm{~mm}$, inter-slice gap $1 \mathrm{~mm}$ ). Each single measurement consisted of 1 offset, 4 baseline and 3 stimulation intervals of $20 \mathrm{~s}$ each; totalling approximately $7 \mathrm{~min}$. additional imaging time for complete somatotopic mapping. During the experiments subjects performed self-paced tongue up and down movements with closed lips, complex finger tapping with sequential finger-to-thumb opposition as well as repetitive toe flexion-extension (without any movements in the ankles) of the side contralateral to the respective lesion $[31,32]$. In the case of tumour-associated paralysis whereby complex finger opposition was not accomplishable, repetitive fist clenching was performed alternatively. Processing and analysis of functional MRI data were carried out with BrainVoyager ${ }^{\circledR}$ (BrainInnovation, Maastricht, Netherlands; http://www.brainvoyager.com), including motion correction, spatial and temporal smoothing and voxelwise calculation of BOLD activation using linear crosscorrelations. Data processing was fully standardised and automated except for semi-automated structural-functional image superposition. BOLD signal characteristics were computed, e.g. correlation of the measured BOLD signal to the applied haemodynamic reference function $(\mathrm{hrf})=\mathrm{r}$ and the relative BOLD signal change $=\Delta S(\%)$. Individual functional data were analyzed using a standardized evaluation routine with a dynamic statistical threshold [30-33]: A minimum cluster size of $36 \mathrm{~mm}^{3}$ was preset as the standard for data evaluation to achieve a precise determination of the anatomical correlates of the different functional activations and also to eliminate very small clusters in the activation maps. At first, a very high statistical threshold value for the correlation ( $\mathrm{r}$ ) between the measured BOLD-signals and the hrf was selected, so that no functional activation was displayed (empty map). This threshold was then continually reduced (dynamic threshold). As a result, the activation with the highest correlation to the hrf that exceeded the cluster size of $36 \mathrm{~mm}^{3}$ was displayed foremost. By further reducing the threshold, activations in other functional areas with a lower correlation between the measured BOLD-signals and the hrf were displayed in hierarchical order. This procedure was continued until activations were identified in all regions of interests (ROI). A minimum threshold of $r=$ 0.4 with $p<0.05$ (Bonferroni corrected) was established as very conservative limit in order to ensure that BOLDsignals were clearly distinguishable from background noise. If no BOLD-activation was displayed in a ROI within this lower limit, this was evaluated as "no activation." Likewise, BOLD signals with a relative change of $\Delta S>5 \%$ were considered bias and not included in the evaluation, as such high-level activations are likely to originate from draining veins [32]. In keeping with our previous studies activations with the highest correlation to the hrf were considered precentral and therefore used as functional landmarks, namely (Fig. 2):

- Cortical foot representation (contralateral to the toes moved)

- Cortical hand representation (contralateral to the fingers moved)

- Cortical tongue representation (bilateral)

\section{Statistical analysis}

Comparison of both diagnostic MRI tools was only feasible with regard to the motor hand area, as it is the only body representation with a distinct anatomical landmark. Statistical evaluation was performed using a Student's t-test to assess a significance of differences and the reliability of localising the motor hand area comparing MRI and fMRI data.

\section{Results}

Localisation of the motor hand area with morphological MRI

According to morphological analyses in regular anatomical proportions of the unaffected hemisphere, the thickness sign and the hand knob were the most reliable anatomical landmarks with a success rate of 99\% (76/77) and 97\% (75/ 77) respectively (see Table 2). In the tumour-affected hemisphere, however, those landmarks could only be identified in $49 \%(38 / 77)$ and $86 \%(66 / 77)$ of the patients. In $14 \%(11 / 77)$ of patients it was not possible to identify the motor hand area at all according to anatomical criteria. The precentral hook as a sagittal pendant to the hand knob was less detectable and in this group did not yield any additional information if the hand knob could not be identified unambiguously. 

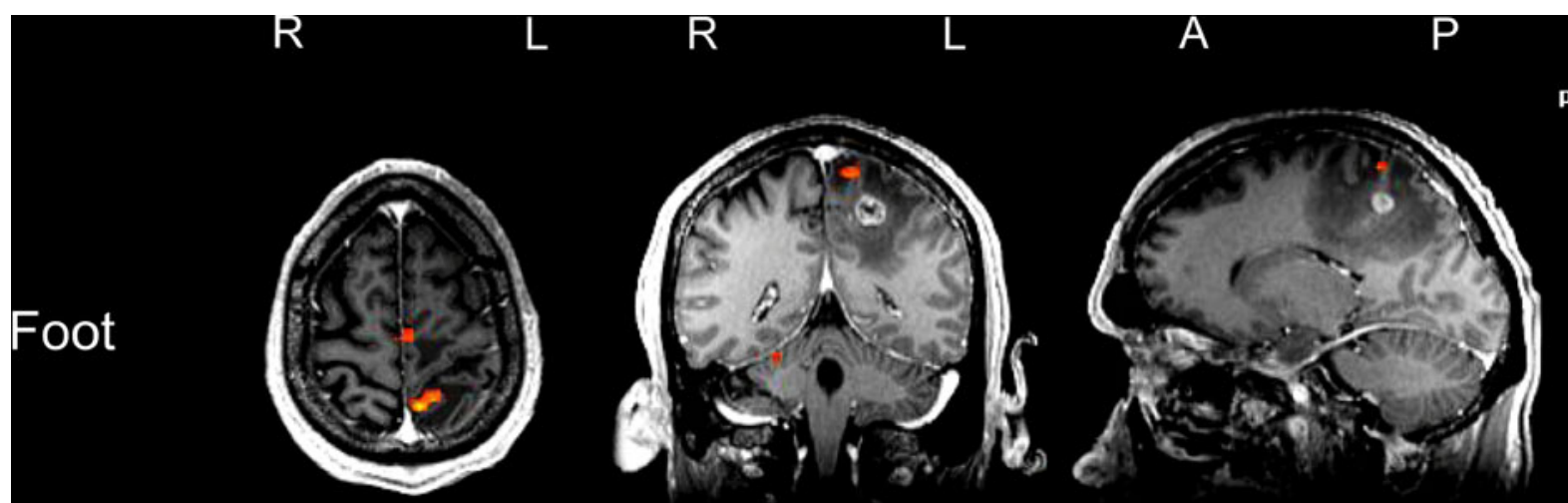

p (cor.) $<0.000$

10.00

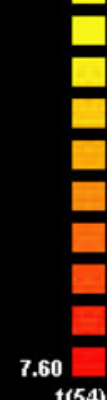

$\mathbf{t}(54)$
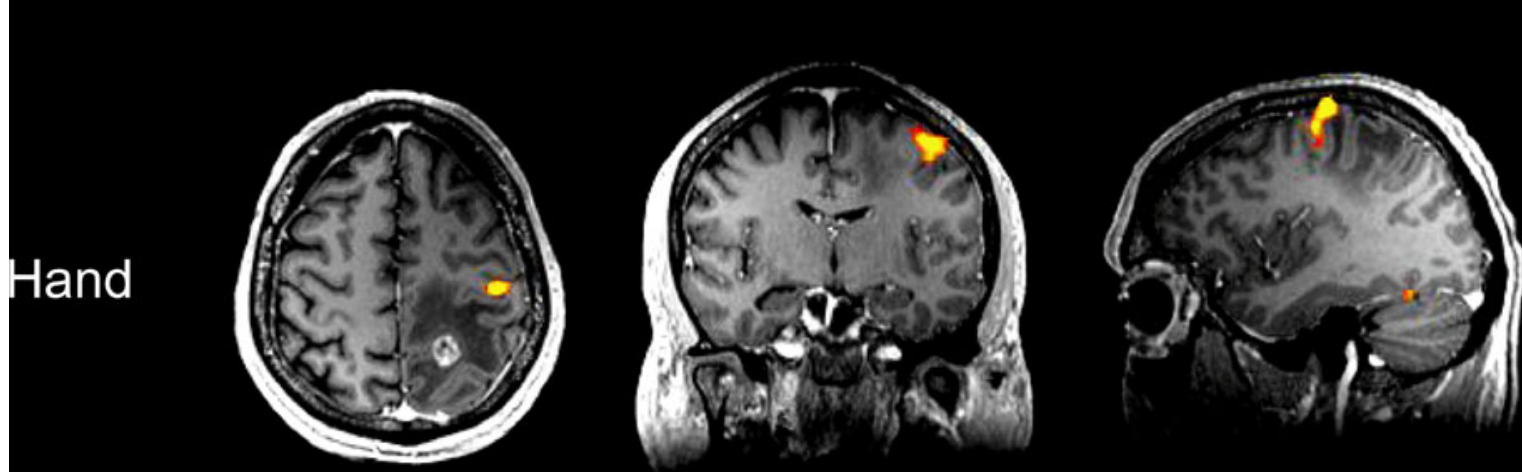

$p($ cor. $)<0.001$

10.00

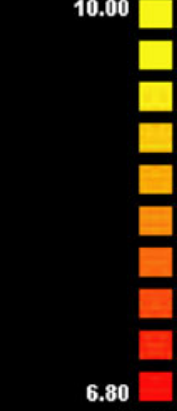

$t(54)$

p $<8.7021 \mathrm{e}-009$

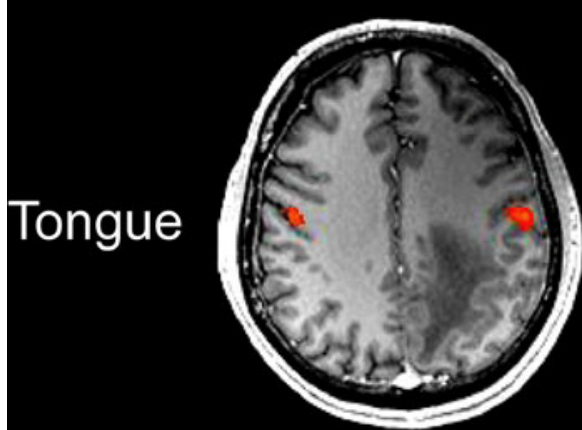

transverse

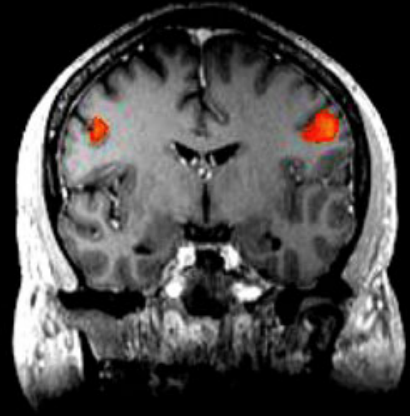

coronal

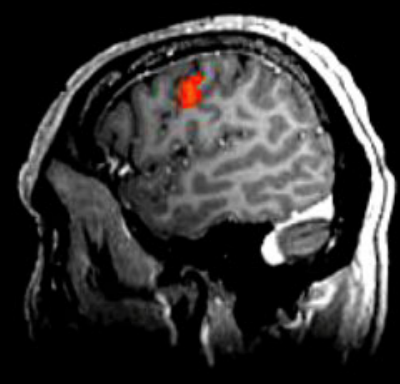

sagittal $p($ cor. $)<0.012$ 10.00

6.00

$t(54)$
Fig. 2 Functional landmarks of the central region (51-year-old female patient with cerebral metastasis of lung adenocarcinoma and impaired fine motor skills of the left hand). Axial (left), coronal (middle) and sagittal (right) sections depict motor areas for foot (top), hand (middle) and tongue (bottom). With morphological criteria alone, localisation of the central region was not feasible due to tumour-associated distortions. Functional landmarks however illustrate how the perifocal oedema but not the contrast-enhancing metastasis itself reaches eloquent areas of the postcentral gyrus. Anterior (A), posterior (P), left $(\mathrm{L})$, right $(\mathrm{R})$
Localisation of motor somatotopy with functional MRI

Functional somatotopic mapping of the motor cortex was conducted in the tumour-affected hemisphere (contralateral movements). Here, the motor hand area could be identified in $99 \%$ of cases (76/77) (Table 3). Although as many as 29 patients suffered from tumour-associated pareses, localisation of the motor hand area was still possible in all but one case.
Thus, additional functional MRI was significantly superior to morphological MRI $(p=0.002)$ regarding the motor hand area localisation. Motor areas of the foot and tongue were investigated in 70 patients and could be robustly identified in $97 \%(68 / 70)$ and $96 \%(67 / 70)$ of patients respectively (Table 3). As reliable morphological landmarks are not available for motor foot and tongue areas, these representations could only be located employing the fMRI technique. 
Table 2 Detection of morphological landmarks in the affected and unaffected hemispheres.

The number of cases is presented in which the classical morphological landmarks for identification of the central region could be detected. In regular anatomical proportions, the thickness sign and hand knob were the most reliable landmarks. However, if mass lesions led to changes in morphological proportions, these landmarks were identified more rarely

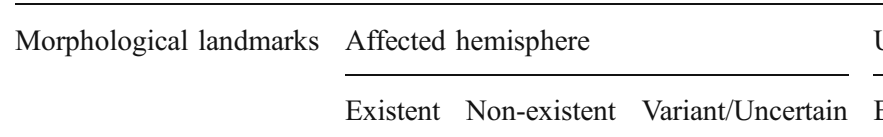

Unaffected hemisphere

Existent Non-existent Variant/Uncertain Existent Non-existent Variant/

Uncertain

Axial:

\begin{tabular}{|c|c|c|c|c|c|c|}
\hline Thickness sign & 38 & 39 & 0 & 76 & 1 & 0 \\
\hline Hand knob & 66 & 11 & 0 & 75 & 0 & 2 \\
\hline Bracket sign & 55 & 20 & 2 & 74 & 1 & 2 \\
\hline \multicolumn{7}{|l|}{ Sagittal: } \\
\hline Hook & 54 & 12 & 11 & 73 & 1 & 3 \\
\hline Pars marginalis & 21 & 54 & 2 & 69 & 0 & 8 \\
\hline Inferior frontal gyrus & 39 & 16 & 22 & 51 & 0 & 26 \\
\hline
\end{tabular}

Diagnostic benefit of presurgical fMRI

After careful consideration of the clinical condition, tumour aetiology and fMRI results the decision for neurosurgical operation was made in $67.5 \%$ (52/ 77) patients. fMRI was used to show the centre of gravity of activation clusters but not to delineate resection borders. In 49 patients complete resection was attempted, whereas 3 patients received only partial resection. The remaining 25 patients who were initially scheduled for surgery did not undergo any operation. Hence, these patients received conservative treatment such as radiation (e.g. arteriovenous angiomas, multiple metastases) and/or chemotherapy (e.g. multiple metastases). In 9 patients this decision was not explicitly based on fMRI results but rather on poor clinical condition or the aetiology of the mass lesion. However, in 16 patients the decision against surgery was mainly based on fMRI results, which provided evidence that major neurological impairments had to be expected after surgery. High risk of surgery-induced deficits was suspected if the centre of activation was localised within or near proximity to destroyed or contrast-enhancing tissue and if the gyrus concerned showed signal alterations on T2-weighted images. Without exception, these 16 tumours were of malignant origin, mainly gliomas of different stages.

By comparing pre- and postoperative outcome of all patients who underwent neurosurgery, 12 patients' clinical status improved after surgery, 15 showed mild or temporary impairment and 4 patients presented with severe postoperative neurological impairment. No change with regard to neurological functions could be observed in 19 patients. In other words, fMRI-based risk assessment before surgery had a high correlation with the clinical outcome achieved and corresponded in $88 \%$ (46 out of 52 post-operative patients) with only minimal deficits or even functional improvement.

\section{Discussion}

In patients with brain tumours of the central region therapeutic decisions strongly depend on preoperative imaging results. It is particularly important to assess to what extent eloquent brain areas are affected by the tumour and if neurological deficits can be expected after neurosurgical tumour removal. Infiltrative, progressive tumour growth is often associated with distortion of anatomical lead structures and therefore inflicting restrictions to morphological imaging techniques. Mostly, patients with such aggressive tumours are not amenable to curative surgical resection, thus making the preservation of important brain functions and life quality a very critical value.

Technical and methodological advances led to increased application of preoperative fMRI in pre-surgical neuroimaging, in particular in large medical centres. To what extent diagnostic benefits can be obtained by presurgical fMRI compared with conventional MR imaging is currently undetermined. To date only studies in small patient groups with non-standardised and heterogeneous protocols have been reported. Nonetheless, a preliminary notion of diagnostic benefits of fMRI over MRI has been suggested by Yetkin et al [28]. Unfortunately, only 3 patients with brain tumours were included in the aforementioned study, none of whom had a lesion with mass effect located directly in the central region, thus raising serious concerns regarding the validity of their results at the time. Similarly, Towle et al [27] compared MRI, fMRI, EEG dipole localisation and direct cortical stimulation in a small group of 5 patients with brain tumours and found that preoperative fMRI reliably identified the central region. Krings et al and Hoeller et al investigated large patient groups with more than 100 patients and concluded that a combination of MRI and fMRI was capable of improving postoperative outcomes of patients and that presurgical fMRI should be 
Table 3 Detection of ipsilesional functional landmarks. The motor hand area in the affected hemisphere was identified in 99\% (76/77). The motor foot and tongue areas were investigated in 70 patients and could be identified in 97\% (68/70) and 96\% (67/70) respectively

\begin{tabular}{lccccc}
\hline Functional landmarks & Existent & Severe paresis & Movement artefacts & Not investigated & Technical error \\
\hline Fingers contralateral & 76 & 1 & 0 & 0 & 0 \\
Toes contralateral & 68 & 1 & 1 & 0 & 7 \\
Tongue & 67 & 0 & 0 & 3 \\
\hline
\end{tabular}

included in standard preoperative neuroimaging protocols [34-36]. However, no direct comparison of MRI and fMRI was conducted nor has it been discussed how far fMRI contributed to therapeutic decision-making. The largest presurgical fMRI study reported in the literature was performed by Hirsch et al on 125 patients partially correlating fMRI results with intraoperative mapping methods [37]. This study reported a correlation of different functional techniques with regard to the localisation of various brain areas. However, resembling other studies previously mentioned, the patient group under investigation was heterogeneous (with a variety of lesions in many different brain areas), no direct clinical outcome was taken into consideration and the benefits of pre-surgical fMRI compared with morphological MRI were not a focus of their study.

The present study of 77 patients was specifically focused on the diagnostic benefit of presurgical fMRI with respect to the localisation of important functional areas adjacent to brain tumours of the central region. Morphological evaluation of regular anatomical proportions in the healthy hemisphere revealed the thickness sign and the hand knob to be the most reliable anatomical landmarks with $99 \%$ and $97 \%$ detection rates in our patient group. In the tumouraffected hemisphere, however, these landmarks could only be identified in $49 \%$ and $86 \%$ respectively; in $14 \%$ of cases the motor hand area could not be localised according to anatomical landmarks.

Somatotopic fMRI mapping yielded robust localisation of motor representations of the hands, feet and tongue; with a success rate of up to $100 \%$ in healthy subjects [12]. In our patient group the central region could be identified in 99\% using optimised fMRI protocols despite several patients suffering from tumour-associated pareses. With regard to the localisation of the motor hand area fMRI proved to be significantly superior $(p=0.002)$ to conventional MRI, while the remaining body representations could only be localised with fMRI. With an additional imaging time of approximately 7 minutes for complete somatotopic mapping, fMRI can be conveniently integrated into presurgical neuroimaging. Furthermore, post-acquisition data processing and interpretation can be easily carried out within the clinical routine if standardised protocols and automated data analysis packages are employed [33, 38]. In the case of severe paralysis, fMRI can be conducted using sensory (passive) stimulation [33, 34, 39], which yields additional information in spite of lower BOLD signal read outs.

Despite the advantages of pre-surgical fMRI presented in our study methodological limitations and potential caveats should not be disregarded. These include movement artefacts, potential superimposition and coregistration errors as well as altered BOLD responses in hypervascularised tumours or arteriovenous malformations [40, 41].

Furthermore, the frequently encountered question of neurosurgeons with regard to the distance that should be maintained between the resection border and functional representation [42] can in our view not (yet) be reliably addressed with fMRI. Obviously, the rate of surgeryinduced neurological deficits correlates inversely with the distance from the tumour-adjacent brain tissue. However, as the threshold for activation signals as well as activation size can be dynamically altered by the investigator and neither a standardised approach nor a multicentre randomised clinical trial addressing this issue exists, using fMRI to delineate resection borders would in our opinion result in operating under a false sense of security and may even endanger the patient.

In agreement with reports on other patient groups, results from our study showed the diagnostic value of preoperative fMRI. In particular those patients whose surgery was called off as a result of presurgical fMRI had the highest benefit. In the present study this group comprised 14 patients $(\sim 18 \%)$.

As fMRI results often influence therapeutic decisionmaking we believe that the additional diagnostic effort of performing fMRI is fully justified and should therefore be routinely implemented in pre-surgical diagnostics. Moreover, better assessment of the spatial relationship between the tumour and the surrounding sensorimotor cortex facilitates the planning and performance of functionpreserving operations, further advocating the inclusion of fMRI into the routine of pre-operative investigations. Hence, presurgical fMRI can substantially contribute to the reduction of therapy-associated morbidity and consequent hospitalisation time. 
Open Access This article is distributed under the terms of the Creative Commons Attribution Noncommercial License which permits any noncommercial use, distribution, and reproduction in any medium, provided the original author(s) and source are credited.

\section{References}

1. Vlieger EJ, Majoie CB, Leenstra S, Den Heeten GJ (2004) Functional magnetic resonance imaging for neurosurgical planning in neurooncology. Eur Radiol 14:1143-1153

2. Naidich TP, Grant JL, Altman N et al (1994) The developing cerebral surface. Preliminary report on the patterns of sulcal and gyral maturation - anatomy, ultrasound, and magnetic resonance imaging. Neuroimaging Clin N Am 4:201-240

3. Rumeau C, Tzourio N, Murayama N et al (1994) Location of hand function in the sensorimotor cortex: MR and functional correlation. AJNR Am J Neuroradiol 15:567-572

4. Achten E, Jackson GD, Cameron JA, Abbott DF, Stella DL, Fabinyi GC (1999) Presurgical evaluation of the motor hand area with functional MR imaging in patients with tumors and dysplastic lesions. Radiology 210:529-538

5. Yousry TA, Schmid UD, Alkadhi H et al (1997) Localization of the motor hand area to a knob on the precentral gyrus. A new landmark. Brain 120:141-157

6. Naidich TP, Blum JT, Firestone MI (2001) The parasagittal line: an anatomic landmark for axial imaging. AJNR Am J Neuroradiol 22:885-895

7. Fesl G, Moriggl B, Schmid UD, Naidich TP, Herholz K, Yousry TA (2003) Inferior central sulcus: variations of anatomy and function on the example of the motor tongue area. Neuroimage 20:601-610

8. Penfield W, Rasmussen T (1950) The cerebral cortex of man. Macmillan Publishing Co, New York, pp 468

9. Wunderlich G, Knorr U, Herzog H, Kiwit JC, Freund HJ, Seitz RJ (1998) Precentral glioma location determines the displacement of cortical hand representation. Neurosurgery 42:18-26

10. Desmurget M, Bonnetblanc F, Duffau H (2007) Contrasting acute and slow-growing lesions: a new door to brain plasticity. Brain 130:898-914

11. Duffau H (2001) Acute functional reorganisation of the human motor cortex during resection of central lesions: a study using intraoperative brain mapping. J Neurol Neurosurg Psychiatry 70:506-513

12. Sunaert S (2006) Presurgical planning for tumor resectioning. J Magn Reson Imaging 23:887-905

13. Tieleman A, Deblaere K, Van Roost D, Van Damme O, Achten E (2009) Preoperative fMRI in tumour surgery. Eur Radiol 19:25232534

14. Stippich C, Mohammed J, Kress B et al (2003) Robust localization and lateralization of human language function: an optimized clinical functional magnetic resonance imaging protocol. Neurosci Lett 346(1-2):109-113

15. Bandettini PA, Jesmanowicz A, Wong EC, Hyde JS (1993) Processing strategies for time-course data sets in functional MRI of the human brain. Magn Reson Med 30:161-173

16. Logothetis NK, Pauls J, Augath M, Trinath T, Oeltermann A (2001) Neurophysiological investigation of the basis of the fMRI signal. Nature 412:150-157

17. Ogawa S, Lee TM, Kay AR, Tank DW (1990) Brain magnetic resonance imaging with contrast dependent on blood oxygenation. Proc Natl Acad Sci USA 87(24):9868-9872

18. Toronov V, Walker S, Gupta R et al (2003) The roles of changes in deoxyhemoglobin concentration and regional cerebral blood volume in the fMRI BOLD signal. Neuroimage 19:1521-1531
19. Majos A, Tybor K, Stefanczyk L, Goraj B (2005) Cortical mapping by functional magnetic resonance imaging in patients with brain tumors. Eur Radiol 15:1148-1158

20. Lehericy S, Duffau H, Cornu P et al (2000) Correspondence between functional magnetic resonance imaging somatotopy and individual brain anatomy of the central region: comparison with intraoperative stimulation in patients with brain tumors. J Neurosurg 92:589-598

21. Roessler K, Donat M, Lanzenberger R et al (2005) Evaluation of preoperative high magnetic field motor functional MRI (3 Tesla) in glioma patients by navigated electrocortical stimulation and postoperative outcome. J Neurol Neurosurg Psychiatry 76:1152-1157

22. Roux FE, Boulanouar K, Lotterie JA, Mejdoubi M, LeSage JP, Berry I (2003) Language functional magnetic resonance imaging in preoperative assessment of language areas: correlation with direct cortical stimulation. Neurosurgery 52:1335-1345

23. Yetkin FZ, Mueller WM, Morris GL et al (1997) Functional MR activation correlated with intraoperative cortical mapping. AJNR Am J Neuroradiol 18:1311-1315

24. Atlas SW, Howard RS 2nd, Maldjian J et al (1996) Functional magnetic resonance imaging of regional brain activity in patients with intracerebral gliomas: findings and implications for clinical management. Neurosurgery 38:329-338

25. De Tiege X, Connelly A, Liegeois F et al (2009) Influence of motor functional magnetic resonance imaging on the surgical management of children and adolescents with symptomatic focal epilepsy. Neurosurgery 64:856-864

26. Lee CC, Ward HA, Sharbrough FW et al (1999) Assessment of functional MR imaging in neurosurgical planning. AJNR Am J Neuroradiol 20:1511-1519

27. Towle VL, Khorasani L, Uftring S et al (2003) Noninvasive identification of human central sulcus: a comparison of gyral morphology, functional MRI, dipole localization, and direct cortical mapping. Neuroimage 19:684-697

28. Yetkin FZ, Papke RA, Mark LP, Daniels DL, Mueller WM, Haughton VM (1995) Location of the sensorimotor cortex: functional and conventional MR compared. AJNR Am J Neuroradiol $16: 2109-2113$

29. Naidich TP, Hof PR, Yousry TA, Yousry I (2001) The motor cortex: anatomic substrates of function. Neuroimaging Clin N Am 11:171-193, vii-viii

30. Stippich C, Ochmann H, Sartor K (2002) Somatotopic mapping of the human primary sensorimotor cortex during motor imagery and motor execution by functional magnetic resonance imaging. Neurosci Lett 331:50-54

31. Stippich C, Romanowski A, Nennig E, Kress B, Sartor K (2005) Timeefficient localization of the human secondary somatosensory cortex by functional magnetic resonance imaging. Neurosci Lett 381:264-268

32. Stippich C, Blatow M, Durst A, Dreyhaupt J, Sartor K (2007) Global activation of primary motor cortex during voluntary movements in man. Neuroimage 34:1227-1237

33. Stippich C, Romanowski A, Nennig E, Kress B, Hahnel S, Sartor K (2004) Fully automated localization of the human primary somatosensory cortex in one minute by functional magnetic resonance imaging. Neurosci Lett 364:90-93

34. Hoeller M, Krings T, Reinges MH, Hans FJ, Gilsbach JM, Thron A (2002) Movement artefacts and MR BOLD signal increase during different paradigms for mapping the sensorimotor cortex. Acta Neurochir (Wien) 144:279-284

35. Krings T, Reinges MH, Erberich S et al (2001) Functional MRI for presurgical planning: problems, artefacts, and solution strategies. J Neurol Neurosurg Psychiatry 70:749-760

36. Krings T, Topper R, Willmes K, Reinges MH, Gilsbach JM, Thron A (2002) Activation in primary and secondary motor areas in patients with CNS neoplasms and weakness. Neurology 58:381-390

37. Hirsch J, Ruge MI, Kim KH et al (2000) An integrated functional magnetic resonance imaging procedure for preoperative mapping 
of cortical areas associated with tactile, motor, language, and visual functions. Neurosurgery 47:711-721

38. Chakraborty A, McEvoy AW (2008) Presurgical functional mapping with functional RI. Curr Opin Neurol 21:446-451

39. Blatow M, Nennig E, Durst A, Sartor K, Stippich C (2007) fMRI reflects functional connectivity of human somatosensory cortex. Neuroimage 37:927-936

40. Carpentier AC, Constable RT, Schlosser MJ et al (2001) Patterns of functional magnetic resonance imaging activation in association with structural lesions in the rolandic region: a classification system. J Neurosurg 94:946-954
41. Ulmer JL, Hacein-Bey L, Mathews VP et al (2004) Lesioninduced pseudo-dominance at functional magnetic resonance imaging: implications for preoperative assessments. Neurosurgery 55:569-579

42. Haberg A, Kvistad KA, Unsgard G, Haraldseth O (2004) Preoperative blood oxygen level-dependent functional magnetic resonance imaging in patients with primary brain tumors: clinical application and outcome. Neurosurgery 54:902-914

43. Stippich C, Blatow M, Krakow K (2007) Presurgical functional MRI in patients with brain tumorsClinical functional MRI. Springer Verlag, pp 90 